\title{
¿Es hora de un cambio de ministro? La supervivencia del gabinete en Colombia 1958-2018
}

\author{
Luis Bernardo MejÍA Guinand* \\ ANDrÉs Felipe Barinas Forero** \\ Michelle Lorena Mora Oviedo***
}

Artículo recibido: 3 de diciembre de 2019

Artículo aprobado: 7 de septiembre de 2020

Doi: https://doi.org/10.12804/revistas.urosario.edu.co/desafios/a.8524

Para citar este artículo: Mejía Guinand, L. B., Barinas Forero, A. F., \& Mora Oviedo, M. L. (2021). ¿Es hora de un cambio de ministro? La supervivencia del gabinete en Colombia 1958-2018. Desafíos, 33(2), 1-31. https://doi.org/10.12804/revistas. urosario.edu.co/desafios/a.8524

\section{Resumen}

Este articulo analiza la formación y supervivencia de los gabinetes presidenciales en Colombia entre 1958 y 2018. Se comparan dos periodos de la historia política del país. Durante el primerperiodo, de 1958 a 1990, Colombia funcionó con un sistema bipartidista. El segundo periodo, de 1991 a la actualidad, se caracteriza por la apertura del régimen político a nuevos partidos y la formación de gobiernos de coalición. Para esto, utilizamos modelos semiparamétricos y regresiones de Cox que nos permiten estimar la supervivencia de 581 nombramientos ministeriales e identificar los predictores de supervivencia en sus cargos a partir tanto de las características individuales de los

\footnotetext{
* Departamento de Ciencia Política, Universidad de los Andes. Correo electrónico: luimejia@uniandes.edu.co. ORCID: https://orcid.org/0000-0003-1339-6262

** Universidad de los Andes. Correo electrónico: af.barinas@uniandes.edu.co. ORCID: https://orcid.org/0000-0003-1413-3923

*** Governance Consultants. Correo electrónico: ml.mora10@uniandes.edu.co. ORCID: https://orcid.org/0000-0001-5329-5381
} 


\title{
2 I Luis Bernardo Mejía Guinand - Andrés Felipe Barinas Forero - Michelle Lorena Mora Oviedo
}

ministros como de las características institucionales del sistema. El estudio muestra que la estabilidad en los cargos de los ministros durante el periodo bipartidista es menor que la duración de los ministros durante la etapa de presidencialismos de coalición.

Palabras clave: gabinete presidencial; supervivencia de ministros; presidencialismo de coalición; Colombia

Clasificación JEL: D72, H19, D78.

\section{Is it Time for a Minister Change? Cabinet Survival in Colombia 1958-2018}

\begin{abstract}
This paper analyzes the formation and survival of presidential cabinets in Colombia between 1958 and 2018. We compare two periods in the country's political history: During the first period, 1958 to 1990, Colombia operated with a two-party system. The second period, 1991 to the present, is characterized by the opening of the political regime to new parties and the formation of coalition governments. For this purpose, we use semi-parametric models and Cox regressions that allow us to estimate the survival of 581 ministerial appointments and identify the predictors of survival in their positions from both individual characteristics of the ministers and the institutional characteristics of the system. The study shows that the stability in the position of ministers during the bipartisan era is lower than the duration of ministers during the period of coalition governments.

Keywords: Presidential cabinet; survival of ministers; coalition presidentialism; Colombia

JEL classification: D72, H19, D78.
\end{abstract}




\section{É hora de uma mudança de ministro? A sobrevivência do gabinete na Colômbia 1958-2018}

\section{Resumo}

Este artigo analisa a formação e a sobrevivência dos gabinetes presidenciais na Colômbia entre 1958 e 2018. Comparam-se dois periodos da história política do pais. Durante o primeiro período, de 1958 a 1990, a Colômbia operou com um sistema bipartidário. O segundo periodo, de 1991 até o presente, é caracterizado pela abertura do regime político a novos partidos e pela formação de governos de coalizão. Para isso, utilizamos modelos semiparamétricos e regressões de Cox que nos permitem estimar a sobrevivência de 581 nomeações ministeriais e identificar os preditores de sobrevivência em seus cargos, com base nas características individuais dos ministros e nas características institucionais do sistema. O estudo mostra que a estabilidade nos cargos de ministros durante o periodo bipartidários é menor do que a duração do mandato de ministros em presidências de coalizão.

Palavras-chave: gabinete presidencial; sobrevivência de ministros; presidencialismo de coalizão; Colômbia

Classificação JEL: D72, H19, D78.

\section{Introducción}

Los presidentes tienen la responsabilidad de plantear los lineamientos estratégicos de las políticas públicas que serán implementadas durante su mandato. Para esto, los mandatarios se apoyan en su equipo de gobierno, principalmente en el gabinete ministerial. Dentro de la Presidencia, los ministros participan activamente en el diseño, formulación e implementación de políticas públicas. De igual forma, influyen en la generación de leyes gracias a su facultad de iniciativa legislativa. En este contexto, una de las primeras tareas que enfrentan los presidentes es diseñar su gabinete.

En los regímenes presidenciales, la conformación del gabinete suele responder a varios factores. Primero, a la necesidad de encontrar a las mejores personas. Individuos con la formación y la experiencia técnica 


\section{4 / Luis Bernardo Mejía Guinand - Andrés Felipe Barinas Forero - Michelle Lorena Mora OViedo}

y política necesaria para llevar a cabo las prioridades del mandatario. Segundo, a la exigencia de representación política del partido del presidente en el gabinete. Tercero, al requerimiento de lograr respaldos políticos regionales y/o gremiales. Esto es, contar con ministros en el gabinete que provengan de regiones del país o representen gremios específicos. Cuarto, a buscar una mejor participación de la mujer y minorías étnicas en los niveles de decisión. Por ejemplo, en algunos países, se dispone que un determinado porcentaje de los altos cargos públicos deben ser ejercidos por mujeres. Por último, pero no necesariamente menos importante, a la prioridad de encontrar a alguien que sea de la entera confianza del presidente.

De igual forma, las características institucionales del sistema político determinan la decisión de un presidente sobre cómo conformar su gabinete. Como lo ha demostrado la literatura (Amorim Neto, 2006; Chasquetti, 2008; Martínez Gallardo, 2014), los presidentes estructuran su equipo de gobierno con la intención de maximizar la supervivencia de las coaliciones legislativas que los apoyan y, de esta manera, mejorar la gobernabilidad. En este cálculo, el mandatario considera variables relevantes como el número efectivo de partidos en el Congreso, el número de asientos del partido en el legislativo y los poderes legislativos formales del presidente (Amorim Neto, 2006).

De acuerdo con Arana Araya (2012), las características de los ministerios y su preponderancia dentro de la estructura de gobierno tienen, también, efecto en la conformación del gabinete. Los ministerios más relevantes suelen trabajar más estrechamente con el presidente y, por lo tanto, los ministros asignados a estas carteras suelen ser más cercanos al primer mandatario.

La asignación de los ministros resulta, pues, esencial, tanto en la búsqueda de gobernabilidad como en la definición e implementación de política pública. Sin embargo, un aspecto importante que debe tenerse en cuenta es cuánto duran los ministros. Esto no solo porque un mandato de corta duración dificulta el logro de resultados sectoriales, que es un indicador de la seriedad con la que el poder ejecutivo trata 
los temas de política pública, sino también porque es un termómetro que permite medir el desarrollo de las coaliciones legislativas.

Así como las características individuales de los ministros (educación, experiencia, género, raza, etc.) y los factores institucionales (soportes del legislativo, coaliciones, sistema de partidos, tipo de ministerio, etc.) inciden en la conformación del gabinete, estas mismas variables son elementos que inciden en la duración en sus cargos. No obstante, dicha duración puede estar determinada por la forma en que se combinan las características individuales con los factores institucionales. Por ejemplo, las características individuales de los ministros, en sistemas bipartidistas, tendrán un efecto de duración diferente en el encargo de los ministros que las características individuales de estos en sistemas multipartidistas. En concreto, los ministros en sistemas bipartidistas tenderían a durar menos en sus cargos que los ministros en presidencialismos de coalición. Para probar esta hipótesis, este artículo analiza la rotación de los gabinetes, al igual que los factores que inciden en la supervivencia de los ministros en Colombia en el período pre-Constitución de 1991 y pos-Constitución de 1991.

Ahora bien, Colombia es un buen estudio de caso para poner a prueba la hipótesis mencionada debido a que durante los últimos 60 años el país ha hecho el tránsito de un sistema bipartidista o uno multipartidista. Desde 1958 hasta 1990 Colombia funcionó con un sistema bipartidista donde las coaliciones de gobierno funcionaban alrededor de las facciones de los partidos y no entre ellos. A partir de 1991, el país abrió su régimen político y más partidos entraron a la escena electoral. Los partidos tradicionales perdieron su poder y hoy en día es muy difícil lograr la presidencia y/o gobernabilidad sin un proceso de coalición entre partidos. Este hecho nos permite comparar el impacto del presidencialismo de coalición en la formación de gabinetes y la supervivencia de ministros bajo un sistema bipartidista y uno multipartidista.

El documento está dividido en siete partes, incluyendo esta introducción. La segunda parte presenta un recuento sobre el estudio de la conformación y duración de los gabinetes en Latinoamérica. La 


\section{6 / Luis Bernardo Mejía Guinand - Andrés Felipe Barinas Forero - Michelle Lorena Mora Oviedo}

tercera parte expone el contexto del estudio. Aquí se discute sobre tres hitos importantes en la historia política del país: el Frente Nacional, la promulgación de una nueva Constitución en 1991 y la reforma constitucional que aprobó la reelección presidencial entre 2006 y 2014. Dentro de este apartado se analiza también la proporcionalidad partidista en el gabinete ministerial y se discute sobre la asignación del gabinete como herramienta que tiene el presidente para negociar con los partidos y el legislativo. En la cuarta parte se muestra una caracterización de los ministros colombianos, aquí se analizan variables como el nivel académico o la región de origen, y su efecto en la duración en la cartera. La quinta sección describe los datos y el tipo de análisis estadístico que se utilizó para estimar la supervivencia de 581 ministros en sus cargos. La sexta parte discute los resultados que evidencian una mayor estabilidad en el gabinete ministerial después de la promulgación de la Constitución de 1991 respecto al período previo comprendido entre 1958 y 1994. El último apartado concluye.

\section{Conformación y supervivencia de gabinetes en el presidencialismo}

En un artículo ya clásico, Scott Mainwaring $(1990,1992)$ señaló las consecuencias negativas que para la estabilidad democrática tenía la combinación de regímenes presidencialistas con sistemas multipartidistas. Según el autor, el presidencialismo multipartidista presenta tres problemas: estimula el conflicto entre las ramas del gobierno, dificulta la conformación de coaliciones de gobierno estables y genera condiciones para la polarización del sistema. Desde entonces, han aparecido un conjunto de estudios especializados en poner a prueba la relación que Mainwaring denomina como la “difícil combinación” entre presidencialismo y multipartidismo (Chasquetti, 2001; Cheibub, 2006).

El postulado central de dichos estudios se enfocó en mostrar cómo los presidentes, con un apoyo minoritario de su partido en el legislativo, necesitaban dedicar un mayor esfuerzo a organizar bases parlamentarias sólidas que les permitieran construir una mayoría estable para gobernar. En otras palabras, formar coaliciones (Amorim Neto, 1998; 
Lanzaro, 2001; Chasquetti, 2001; Jones, 2005; Cheibub, 2006, Payne, 2007). En términos generales, cuanto más alto es el número de partidos, más alta es la probabilidad de que aparezcan presidencialismos minoritarios que requieren formar gobiernos de coalición (Albala, 2016). De acuerdo con Amorim Neto (2006), los presidentes que quieren tramitar sus proyectos de gobierno a través de leyes tienden a nombrar como ministros a líderes partidarios, quienes les ayudan a conseguir las mayorías necesarias en el Congreso. El incentivo de estos partidos de unirse al gobierno es influir en la formulación de políticas y controlar su implementación (Figueiredo \& Limongi, 2008).

Otros trabajos han descrito cómo el intercambio de puestos en el gabinete por votos en el Parlamento no necesariamente refleja la prioridad en la búsqueda de gobernabilidad del presidente. Por ejemplo, María Ollier y Pablo Palumbo (2016), en un estudio de caso sobre la formación de gabinetes en Argentina, encuentran que la relación entre el nombramiento de ministros y las relaciones partidistas en el Parlamento no son muy fuertes, llegando a ser incluso inexistentes. Lo anterior incentiva al presidente a buscar apoyo en otro tipo de actores, como las empresas o los sindicatos. De acuerdo con Ollier y Palumbo, la baja institucionalización de los partidos políticos y la centralidad presidencial, combinadas con el contexto en el cual asume y transcurre el mandato de un jefe de Estado, definen la formación del gabinete.

Existe también acuerdo en la literatura en que los presidentes nombran o realizan cambios de ministros como una estrategia para adaptarse a un entorno cambiante y así poder hacer frente a diferentes crisis y conflictos (Camerlo \& Pérez Liñán, 2015; Martínez Gallardo, 2014). Por ejemplo, los escándalos políticos aumentan el riesgo de rotación de ministros, pero sus efectos están condicionados por consideraciones estratégicas y calendarios electorales. Conforme con Camerlo y Pérez Liñán (2015), si los presidentes pueden y aspiran a la reelección, estarían dispuestos a destituir a los ministros que estén envueltos en escándalos mediáticos al comienzo de sus mandatos. Esto porque aún hay tiempo para limpiar la imagen de la administración. En la misma dirección, los presidentes estarían dispuestos a destituir a los 


\section{8 / Luis Bernardo Mejía Guinand - Andrés Felipe Barinas Forero - Michelle Lorena Mora OViedo}

ministros que enfrentan protestas masivas, en contra de la política de su sector, pero esta vez tarde en sus mandatos. Esto ocurriría porque los presidentes carecen de tiempo suficiente para convencer a los votantes de que la política sectorial puede ser exitosa.

Contrario a lo anterior, sugieren Camerlo y Pérez Liñán (2015), los presidentes que enfrentan límites en su período de mandato despedirán a los ministros enfrentados a protestas sociales, en contra de la política sectorial a su cargo, desde los primeros meses de la administración. En cambio, los presidentes mantendrán en el cargo a los ministros contaminados por escándalos mediáticos hasta mediados o finales de su mandato.

De forma adicional, la literatura sobre presidencialismo de coalición en Latinoamérica ha avanzado, a partir de casos de estudio puntuales, especialmente en los países del Cono Sur, en ofrecer explicaciones sobre los determinantes de la supervivencia de los ministros del gabinete. Por ejemplo, para el caso chileno, Bastián González y Alejandro Olivares (2016) analizaron la rotación de los gabinetes y los factores que inciden en la supervivencia de 180 ministros pertenecientes a los gobiernos de la concertación (1990 a 2010). Los autores identifican que existen predictores asociados a las características individuales de los ministros y que tanto las crisis económicas como los escándalos de corrupción influyen en la supervivencia ministerial. Finalmente, Daniel Chasquetti, Daniel Buquet y Antonio Cardarello (2013) estudiaron la formación y cambio de gabinetes presidenciales en Uruguay durante el período 1985-2010. El estudio mostró que los presidentes uruguayos designan preferentemente políticos con filiación partidaria y que los ministerios más importantes (Economía y Finanzas, Relaciones Exteriores, Interior y Defensa Nacional) son asignados a políticos pertenecientes al partido del presidente. Los autores resaltan que la supervivencia de los ministros en sus cargos responde a las lógicas de funcionamiento de las coaliciones o a contingencias vinculadas con decisiones personales de los individuos. 


\section{Del Frente Nacional a la reelección presidencial: el contexto del estudio}

Para entender la rotación de los gabinetes, así como los factores que inciden en la supervivencia de los ministros en Colombia, es importante considerar tres hitos importantes en la historia política del país: el Frente Nacional, la promulgación de una nueva Constitución en 1991 y la reforma constitucional que aprobó la reelección presidencial entre 2006 y 2014.

El Frente Nacional (1958-1974) fue un pacto político diseñado por los dos partidos dominantes (Liberal y Conservador) para poner fin tanto a la violencia bipartidista que el país había experimentado hasta entonces como a la dictadura militar de cuatro años que resultó de dicho conflicto (Hartlyn, 1988; Archer \& Shugart, 1997). Las principales disposiciones del Frente Nacional se centraron en: i) alternar la Presidencia de la República entre los dos partidos. El período presidencial se mantuvo de cuatro años y comenzó el Partido Liberal con el presidente Alberto Lleras Camargo (1958-1962); y ii) compartir las responsabilidades gubernamentales al tener representación equitativa (50\%) de ambos partidos en el Congreso, el gabinete, las oficinas gubernamentales, los puestos del Tribunal Supremo, las asambleas departamentales y los consejos municipales.

Este pacto político fue apoyado por todas las facciones de la clase dominante, los industriales, los banqueros y los comerciantes (Hartlyn, 1988). Además, el Frente Nacional fue legitimado a través de un referéndum en el que se preguntó a los ciudadanos colombianos sobre su aceptación del pacto. Tras su aprobación el $1^{\circ}$ de diciembre de 1957, la estructura política del Frente Nacional se estableció por un período de 16 años. Si bien formalmente el Frente Nacional terminó en 1974, el régimen político continuó cerrado a la participación política de otros partidos o movimientos diferentes a los tradicionales, Liberal y Conservador, hasta el año 1991. Este bloqueo político engendró un sistema de negociación entre dos actores que terminó con la consolidación de una distribución ministerial casi equitativa entre el Partido Liberal y el Partido Conservador, como se ilustra en la figura 1. 
10 / Luis Bernardo Mejía Guinand - Andrés Felipe Barinas Forero Michelle Lorena Mora OViedo

Figura 1. Número de ministros pertenecientes a la coalición: antes de 1990

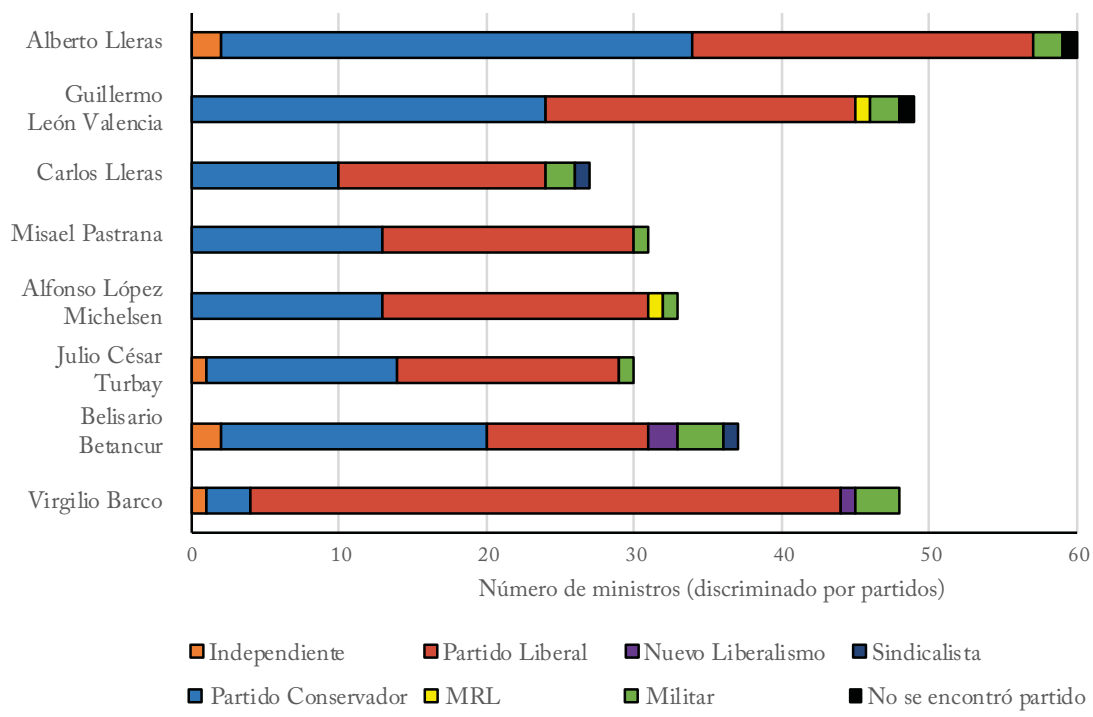

Fuente: elaboración de los autores.

La promulgación de la nueva Constitución Política de Colombia en el año 1991 fue el resultado de la exacerbación de las tensiones políticas y sociales de finales de la década de 1980. El control bipartidista en el que el país había vivido por décadas y la exclusión de movimientos distintos a los tradicionales llevó a amplios segmentos de la población a reclamar mayor participación e inclusión política. De igual forma, las regiones demandaban del centro una mayor descentralización y autonomía en sus decisiones.

De acuerdo con Cárdenas y Pachón (2010), la Carta Política aprobada en 1991 se convirtió en una válvula de escape que permitió al sistema institucional asimilar algunas de las presiones, tanto de orden social como político, que se habían acumulado a lo largo de las décadas anteriores. Esta apertura del régimen político se tradujo en la eliminación de las barreras para la participación política y en la aparición de nuevos movimientos y partidos políticos distintos de los tradicionales. Como resultado, el número efectivo de partidos aumentó, la figura 2 muestra dicha situación. El número efectivo de partidos en 
Colombia pasó de 2,2 en 1990 a 6,3 en 2018 para el Senado, y de 2,2 en 1990 a 7,4 en 2018 para la Cámara.

Figura 2. Número efectivo de partidos en Colombia (1990-2018)

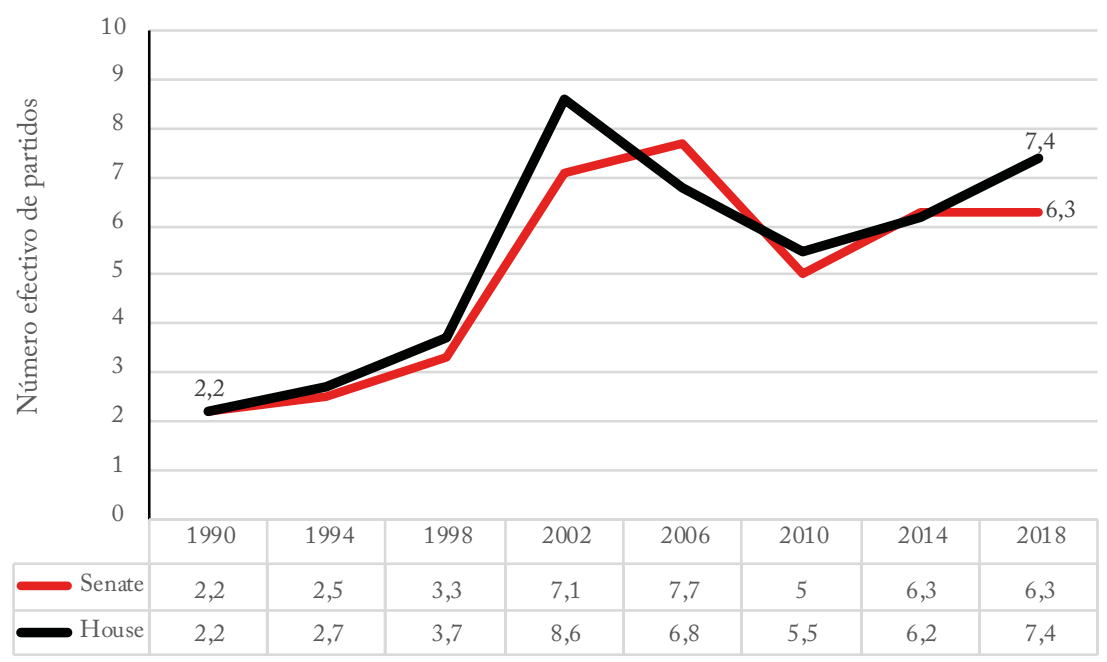

Fuente: elaboración de los autores basados en datos de Congreso Visible.

En la misma dirección, la figura 3 expone la composición partidaria de los gabinetes posteriores a 1990. Aquí se observa el detrimento de la participación de los partidos tradicionales, Liberal y Conservador, en los gabinetes presidenciales en contraste con el aumento de la participación de nuevos partidos. 
12 / Luis Bernardo Mejía Guinand - Andrés Felipe Barinas Forero -

Michelle Lorena Mora Oviedo

Figura 3. Número de ministros pertenecientes a la coalición 1990-2018

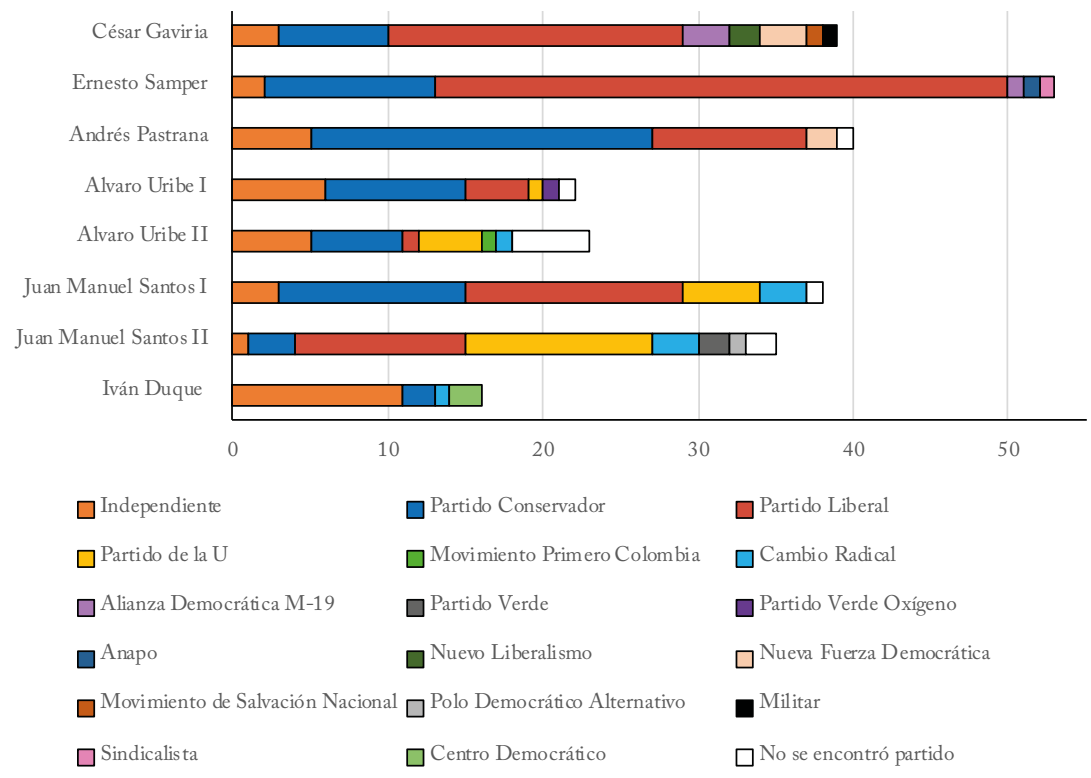

Fuente: elaboración de los autores.

Por último, el Acto Legislativo 2 de 2004 introdujo la figura de la reelección presidencial inmediata en Colombia. Esta permitió que quienes hubiesen ejercido la Presidencia de la República permanecieran en su cargo durante un segundo período si mediante el voto así lo decidieran los ciudadanos. Bajo esta reforma constitucional fueron reelectos los presidentes Álvaro Uribe Vélez (2006) y Juan Manuel Santos (2014). La reelección presidencial permitió que los candidatos presidentes desarrollaran una estrategia legislativa, acorde al calendario electoral, a través de la cual se formaron coaliciones electorales que resultaron, posteriormente, en coaliciones de gobierno. Lo anterior se reflejó en la rotación ministerial preelecciones y en la conformación de los gabinetes de los gobiernos reelectos. En 2015, la figura de la reelección presidencial fue eliminada por el Congreso.

Como se observa en la figura 4, durante los mandatos del Frente Nacional (1958-1974), los partidos Liberal y Conservador, que asumen el rol de partido de gobierno o de coalición según el período, manejan entre el $80 \%$ y el $90 \%$ de las asignaciones de ministros. 
En contraste, en los gobiernos de Uribe (2002-2006 y 2006-2010) y Santos (2010-2014 y 2014-2018), el partido del presidente tiene una participación mínima en el gabinete comparado con la participación de los partidos de la coalición de gobierno. Esto significa que los presidentes, cuyos partidos carecen de una mayoría en el Congreso, obtienen el respaldo de los miembros de otros partidos mediante nombramientos en el gabinete.

\section{Figura 4. Cantidad de ministros según su pertenencia a la coalición}

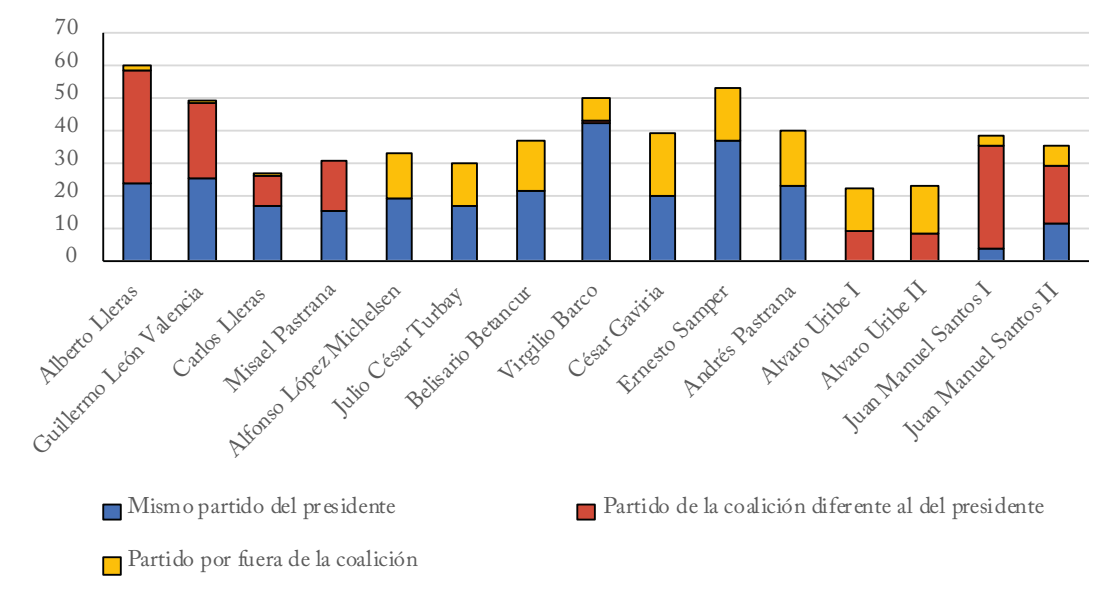

Fuente: elaboración de los autores.

A lo largo de los tres períodos señalados, los gabinetes se constituyeron en una de las herramientas con las que cuenta el presidente para asegurar la formación de coaliciones políticas y lograr la gobernabilidad del mandatario. Esto se puede observar al analizar la proporcionalidad partidista entre el gabinete ministerial y el contingente legislativo. Para esto, se hace uso del Índice de Congruencia Partidista del Gabinete (ICPG) propuesto por Amorim Neto (1998). Este índice hace referencia a la relación entre la representación partidista en la distribución de carteras del gabinete y el peso legislativo de los partidos representados en el Congreso. El índice toma valores entre 0 y 1 , donde a mayor representación de la distribución partidista del legislativo en el gabinete ministerial adquirirá valores más cercanos a 1. Por otro lado, tomará valores más cercanos a 0 si el gabinete no representa la distribución partidista en el Congreso. 
14 / Luis Bernardo Mejía Guinand - Andrés Felipe Barinas Forero Michelle Lorena Mora Oviedo

Como lo evidencia la tabla 1, el promedio del ICPG para las 16 administraciones analizadas es de 0,83 . Esto significa que en promedio los gabinetes representan un porcentaje alto de la composición del legislativo. Particularmente se observa que las administraciones anteriores a 1990 presentan valores más altos del índice en comparación con las administraciones posteriores a 1990. Este fenómeno se explica gracias al régimen político bipartidista que existía antes de los años noventa y al arreglo del Frente Nacional. Al darse la apertura política en la década de los noventa, el número de actores políticos se incrementó no solo en la rama legislativa, sino también en los gabinetes ministeriales. Por lo anterior, es posible afirmar que la construcción de un gabinete que represente las proporciones de participación partidista del Congreso se volvió más compleja, dada la heterogeneidad de los actores y la importancia de ministros independientes sin ninguna afiliación partidista que disminuyen el valor del ICPG.

Tabla 1. Índice de Congruencia Partidista del Gabinete en Colombia

\begin{tabular}{|l|l|}
\hline \multicolumn{1}{|c|}{ Administración } & ICPG \\
\hline Alberto Lleras Camargo & 0,99 \\
\hline Guillermo León Valencia & 0,91 \\
\hline Carlos Lleras Restrepo & 0,94 \\
\hline Misael Pastrana Borrero & 0,84 \\
\hline Alfonso López Michelsen & 0,93 \\
\hline Julio César Turbay Ayala & 0,96 \\
\hline Belisario Betancur Cuartas & 0,85 \\
\hline Virgilio Barco Vargas & 0,67 \\
\hline César Gaviria Trujillo & 0,86 \\
\hline Ernesto Samper Pizano & 0,88 \\
\hline Andrés Pastrana Arango & 0,73 \\
\hline Álvaro Uribe Vélez I & 0,74 \\
\hline Álvaro Uribe Vélez II & 0,80 \\
\hline Juan Manuel Santos I & 0,76 \\
\hline Juan Manuel Santos II & 0,82 \\
\hline Iván Duque & 0,63 \\
\hline
\end{tabular}

Fuente: elaboración de los autores. 


\section{Duración y características individuales de los ministros colombianos}

Entre los años 1958 y 2018, Colombia ha contado con 16 períodos presidenciales ininterrumpidos con una duración constitucional de cuatro años cada uno. El total de ministros que sirvieron a estas administraciones es de 581. Estos ministros han estado a cargo del diseño y la implementación de políticas, planes, programas y proyectos sectoriales. También ha sido su responsabilidad formular y presentar al legislativo distintos proyectos de ley atenientes al área de política de sus despachos. Por último, los ministros se han responsabilizado de la regulación técnica del área de política a la que pertenecen.

Una característica importante en la formación de gabinetes corresponde a la estabilidad de los ministros en su cargo. Como se observa en la figura 5, la permanencia de los ministros en su puesto ha venido en aumento. Mientras que durante el período correspondiente al Frente Nacional (1958-1974) las carteras tenían una rotación promedio de 3,03 ministros por mandato presidencial, la rotación media durante las últimas cuatro administraciones es de 2,33 ministros por cartera. Existe una mayor estabilidad para los gobiernos después de 1990, los cuales rotaron el $25 \%$ de su gabinete a los dos años de su mandato, mientras que los gobiernos comprendidos entre 1958 y 1990 lo hicieron al año. 
Figura 5. Promedio de ministros por cartera

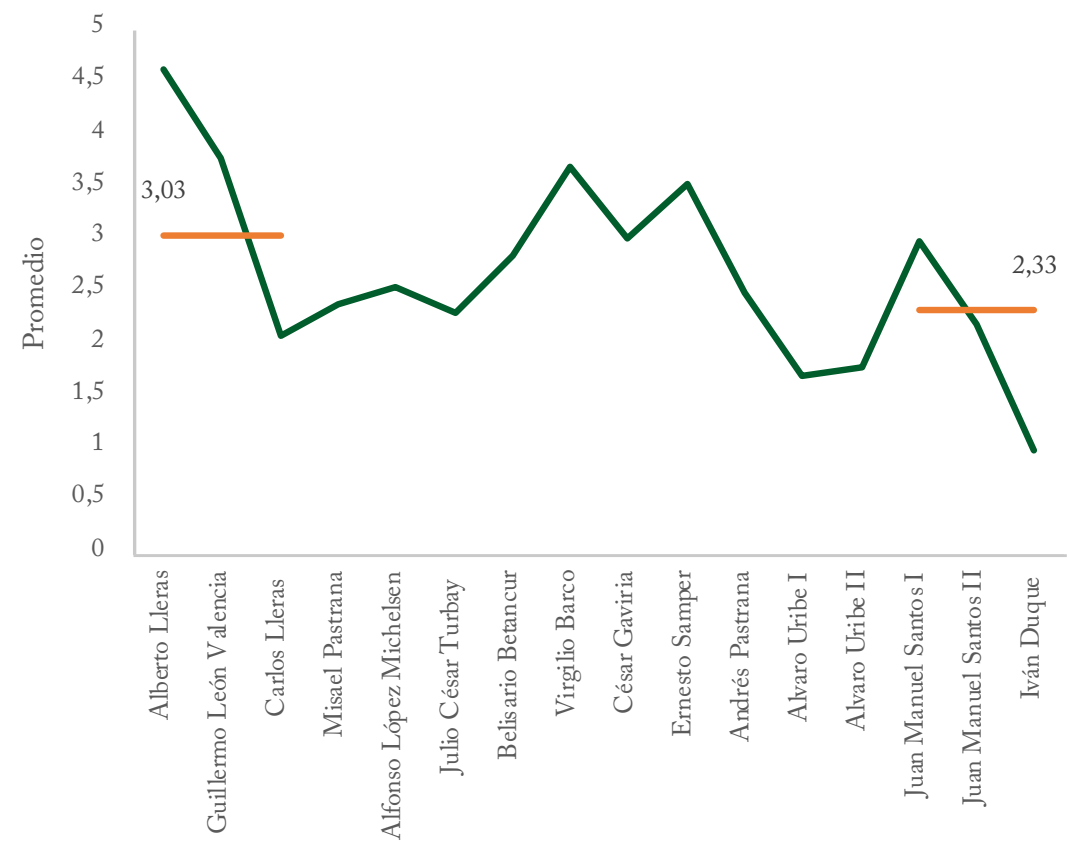

Administración

Fuente: elaboración de los autores.

$\mathrm{Al}$ analizar la duración de los ministros por cartera se puede evidenciar que el ministerio más sostenido es el de relaciones internacionales, con un promedio de 695 días. Por el contrario, los ministerios en los que duran menos los ministros son en los de vivienda, trabajo y salud pública, con un promedio menor a 400 días. Al discriminar por género se observa que las mujeres duran más días en el gabinete que los hombres. El promedio, en días, en el gabinete para las mujeres es de 636, mientras que para los hombres es de 502.

El nivel educativo de las personas que ocupan las carteras ministeriales ha venido en ascenso. Mientras que para la década de 1960 el $64,2 \%$ de los ministros solo tenían formación de pregrado, para la segunda década de 2000, los ministros con especialización, maestría o doctorado representan alrededor del 89,6\%. Además, como se muestra en la tabla 2 , los ministros con maestría son los que más duran 
en el cargo con 634 días en promedio. En términos de profesión, se tiene que el 50,27\% de los ministros nombrados son abogados de profesión y duran en promedio 496 días, el 15,5\% de los ministros son economistas y duran 584 días en promedio, siendo las dos carreras más estudiadas por los ministros. Los ministros con el nivel de educación más alto, doctorado, han asumido carteras estratégicas para el desarrollo del país, como hacienda y crédito público, salud pública, vivienda y agricultura. Sin embargo, la única cartera que es intensiva en ministros con este nivel de estudios es la de hacienda y crédito público.

Los ministros colombianos provienen, principalmente, de la universidad privada. De los ministros que cursaron sus estudios de pregrado en Colombia, se tiene que el $64,7 \%$ ha estudiado en universidades privadas, mientras que el $35,2 \%$ lo ha hecho en universidades públicas. Las universidades de procedencia de estos funcionarios son, en su orden, la Pontificia Universidad Javeriana, con el 21,7\% de los ministros; seguida por la Universidad Nacional de Colombia, con el 17\%; la Universidad de los Andes, con el 12,5\%; la Universidad del Rosario, con el 5,4\%; y la Universidad de Antioquia, también con el 5,4\%. Solamente el $6,9 \%$ de los ministros ha realizado su pregrado en una universidad extranjera.

Al observar la composición regional de los gabinetes, y como se ilustra en la tabla 2, se encuentra que la región Andina es el territorio que mayor número de ministros provee $(63,71 \%)$ y tienen un promedio de duración en el cargo de 540 días; seguida por la región Caribe $(18 \%)$, con una duración promedio de 389 días; la región Pacífica $(15,7 \%)$, con un promedio de 481 días; y, por último, la región Orinoquía-Amazonía, cada una con una participación del 0,36\%, con duraciones de 291 y 367 días promedio. Al desagregar la región Andina, se tiene que Bogotá es el ente territorial con mayor participación en los gabinetes ministeriales en términos relativos y absolutos $(33 \%)$, seguida por Antioquia (21,7\%) y Santander (10,2\%). 
18 / Luis Bernardo Mejía Guinand - Andrés Felipe Barinas Forero Michelle Lorena Mora OViedo

Tabla 2. Promedio de duración de días por nivel académico y por región

\begin{tabular}{|l|c|c|c|c|c|}
\hline \multicolumn{1}{|c|}{$\begin{array}{c}\text { Nivel } \\
\text { académico }\end{array}$} & N & $\begin{array}{c}\text { Promedio } \\
\text { de días }\end{array}$ & SD & Mín. & Máx. \\
\hline Ninguno & 2 & 138 & 53,74 & 100 & 176 \\
\hline Primaria & 2 & 508,5 & 443,356 & 195 & 822 \\
\hline Bachillerato & 6 & 534,833 & 468,415 & 236 & 1461 \\
\hline Pregrado & 238 & 478,164 & 311,249 & 2 & 1461 \\
\hline Especialización & 137 & 498,343 & 359,525 & 2 & 1461 \\
\hline Maestría & 92 & 634,087 & 405,524 & 18 & 1461 \\
\hline Doctorado & 54 & 567,981 & 314,23 & 45 & 1461 \\
\hline Militar & 15 & 574,733 & 400,595 & 232 & 1461 \\
\hline Región & & & & & \\
\hline Andina & 357 & 540,759 & 361,118 & 2 & 1461 \\
\hline Caribe & 99 & 489,919 & 291,464 & 25 & 1461 \\
\hline Pacífica & 89 & 481,562 & 362,519 & 15 & 1461 \\
\hline Orinoquía & 2 & 291,5 & 45,962 & 259 & 324 \\
\hline Amazonía & 2 & 367 & 91,924 & 302 & 432 \\
\hline Extranjero & 2 & 910,5 & 778,525 & 360 & 1461 \\
\hline
\end{tabular}

Fuente: elaboración de los autores.

\section{Datos y estrategia empírica}

Para poner a prueba nuestra hipótesis, utilizamos la base de datos original Presidential Cabinets Project (Prescab) (Mejía Guinand \& Botero, 2019). Esta base contiene información sobre 581 ministros colombianos a lo largo de 16 períodos presidenciales. En concreto, la base reúne los siguientes datos: i) perfiles. Aquí se detallan las características individuales de los ministros, tales como género, edad al momento de asumir el cargo, cartera, profesión, nivel de formación, universidad en la que estudió, región a la que pertenece y vínculos familiares; ii) partidismo. Aquí se registra la militancia, simpatía o no pertenencia de los ministros a un partido político; iii) trayectoria. Este criterio identifica los cargos públicos y/o privados que los ministros han tenido antes y/o después de ocupar el encargo; iv) diseño de gabinete. Describe la conformación partidaria de los gabinetes colombianos entre 1958 y 2018. La información para construir la 
base de datos se recopiló de informes de noticias, sitios web, entrevistas personales y biografías. La base de datos es de acceso público, a través de Harvard Dataverse, y está disponible en la página web del Presidential Cabinets Project (https://www.presidentialcabinets.org/).

Con el fin de estimar la supervivencia de 581 nombramientos ministeriales e identificar los predictores de supervivencia en sus cargos, llevamos a cabo un análisis de supervivencia. Este es un tipo de análisis estadístico que permite determinar el tiempo esperado de duración de una acción hasta que un evento particular ocurra. En este estudio empleamos doce variables independientes que capturan diferencias en características individuales de los ministros.

Para el caso estudiado se entiende como 'acción' a la función de ejercer como ministro durante un número de días determinado. A su vez, el fracaso se entiende como la remoción del cargo antes de la culminación del período presidencial. Se contempla la existencia de ministros que no son removidos durante el mandato, sino que son removidos del cargo cuando culmina el período presidencial, es decir que salen del ejecutivo en compañía del mandatario actual. A este fenómeno se le denomina censura. Dada la existencia de estos datos, es necesario emplear estimadores no paramétricos de KaplanMeier (1958) que contemplen censuras sobre la variable fracaso sin afectar la estimación de parámetros de supervivencia o de riesgo en la duración de los ministros. Emplear otros métodos de estimación paramétricos como mínimos cuadrados ordinarios (MCO) sobre la variable de tiempo de los ministros en el cargo podría hacer que los estimadores fuesen inconsistentes.

Adicionalmente, se emplea la promulgación de la Constitución de 1991 como punto de corte comparativo entre períodos de bipartidismo y períodos de gobiernos de coalición, ya que, como se expuso antes, la participación partidaria en el ejecutivo aumentó después de este cambio constitucional. Con la nueva Constitución la arena política fue más competitiva, el número efectivo de partidos se incrementó y el número de partidos dentro de los gabinetes también creció, 


\section{0 / Luis Bernardo Mejía Guinand - Andrés Felipe Barinas Forero - Michelle Lorena Mora Oviedo}

haciendo que las coaliciones fuesen fundamentales para la gobernabilidad. Para ver las diferencias en la duración de los ministros, se realizó una representación gráfica de los estimadores no paramétricos de Kaplan-Meier en los períodos previo y posterior a la expedición de la Constitución de 1991. La figura 6 contempla estos resultados.

Con el ánimo de determinar cuáles son las variables que explican mayores o menores niveles de riesgo de que un ministro sea removido, se emplean dos modelos de regresión de Cox $(1972,1975)$. Cada modelo se estima para cada muestra temporal, una estimación para la supervivencia de los ministros que ejercieron previo a la Constitución de 1991 y la otra estimación para los ministros que ejercieron después de la Constitución de 1991. Los modelos de regresión de Cox, también conocidos como modelos de riesgos proporcionales, tienen como objetivo plantear un modelo de regresión para el riesgo en función de un conjunto de covariables, predictores o variables independientes que permitan estimar su efecto sobre la probabilidad de fracaso. En este sentido, se utilizan las características individuales de los ministros y otras variables institucionales para identificar su incidencia dentro del tiempo que permanecen en el cargo. Se verifica el cumplimiento del supuesto de riesgos proporcionales para evitar que los estimadores sean sobreestimados y sesgados. Las estimaciones del modelo de Cox $(1972,1975)$ cuando hay presencia de eventos colindantes y datos censurados (como en el caso de análisis de supervivencia de ministros) son problemáticas, No obstante, estas limitaciones pueden ser solucionadas mediante las aproximaciones de máxima verosimilitud de Efron (1977). Por este motivo, los resultados presentados a continuación fueron estimados empleando la aproximación de máxima verosimilitud de Efron (1977).

\section{Resultados}

Los resultados de las curvas de Kaplan-Meier estimadas para los períodos pre y pos-Constitución de 1991 se encuentran en la figura 6. 
Allí, es posible diferenciar el grado de rotación y/o estabilidad ministerial entre ambos períodos. En particular, se evidencia mayor estabilidad en el gabinete ministerial después de la promulgación de la Constitución de 1991 respecto al período previo comprendido entre 1958 y 1994. Durante los períodos de sistemas bipartidistas solo 6 de cada 10 ministros duraba 500 días en el cargo, mientras que en los períodos de gobiernos de coalición se tiene que 8 de cada 10 ministros dura 500 días en el cargo. De igual forma, durante el sistema bipartidista se tenía que solo el 30\% de los ministros duraban más de 1000 días en el cargo, mientras que en los sistemas de coalición se tiene una tasa de supervivencia cercana al $60 \%$.

De igual manera, ambas curvas indican la existencia de dos momentos de relativa estabilidad en el gabinete, es decir, momentos en los que hay menor probabilidad de que los ministros sean removidos: el primero está comprendido entre los días 500 y 600; el segundo, para el período previo a la Constitución, se encuentra entre el día 900 hasta el día 1500, mientras que, para el período posterior a la Constitución, está entre el día 800 y 1500. Como se muestra con el intervalo de confianza al 95\%, las diferencias en la duración de días entre períodos son significativas después de los primeros 200 días en el cargo. 


\section{I Luis Bernardo Mejía Guinand - Andrés Felipe Barinas Forero - Michelle Lorena Mora OVIEdo}

Figura 6. Estimación de supervivencia ministerial en días del mandato transcurridos (curvas de Kaplan-Meier)

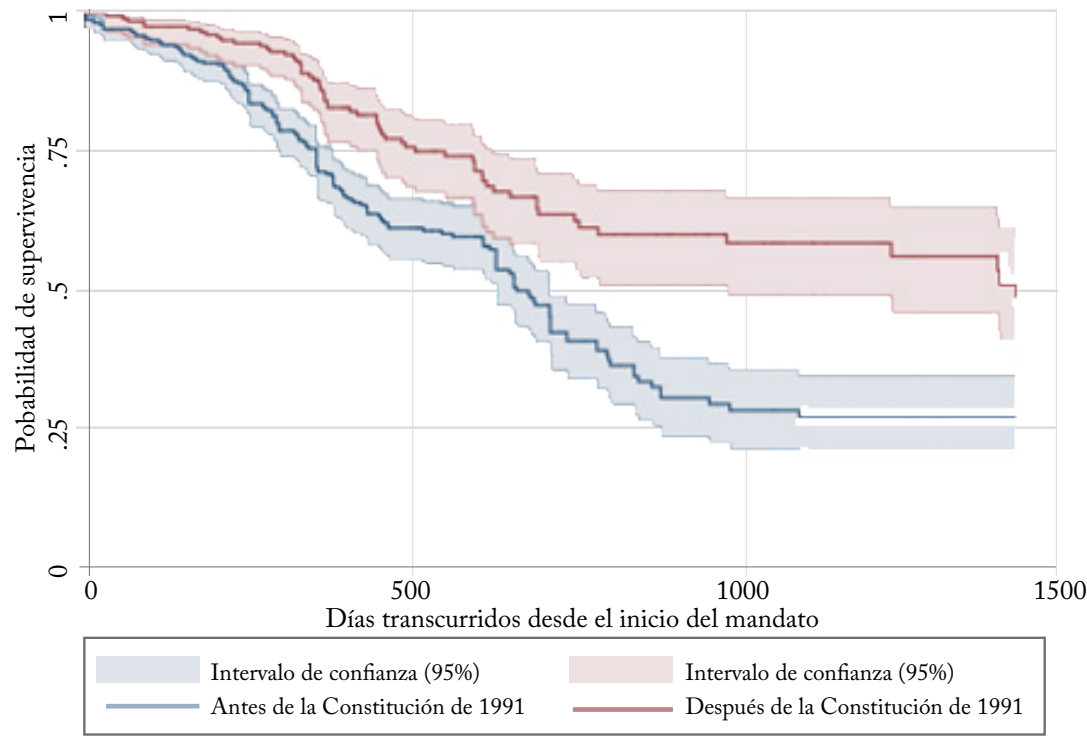

Fuente: elaboración de los autores.

Es posible confirmar esta tendencia diferenciada entre ambos períodos a través de las tasas de incidencia y la exposición al riesgo de rotación ministerial presentadas en la tabla 3. La tasa de incidencia corresponde al nivel de "mortalidad política" de los ministros (González Bustamante \& Olivares, 2016), de manera que, en el período previo a la Constitución de 1991 la tasa de mortalidad era del 34,5\%, valor que casi duplica la tasa en el período posterior a la promulgación de la Constitución, que es del 17,5\%. Además, se reporta que los ministros antes de 1991 presentan cerca de 466 días en riesgo, ${ }^{1}$ mientras que los ministros en el período pos-Constitución de 1991 tienen menos días en riesgo, con 352.

\footnotetext{
1 Los días en riesgo corresponden a un cálculo con base en la cantidad de ministros en cada período estudiado y su tiempo de duración en el gabinete.
} 
Tabla 3. Tasas de incidencia y exposición al riesgo de rotación ministerial

\begin{tabular}{|l|l|l|l|}
\hline \multicolumn{1}{|c|}{ Período } & \multicolumn{1}{c|}{ Días en riesgo } & \multicolumn{1}{|c|}{ Fracasos } & \multicolumn{1}{c|}{ Tasa de incidencia $^{2}$} \\
\hline Antes de 1991 & 466,642 & 161 & 0,34501 \\
\hline Después de 1991 & 352,908 & 62 & 0,17568 \\
\hline Total & 299,341 & 223 & 0,27210 \\
\hline
\end{tabular}

Fuente: elaboración de los autores.

Gráficamente es posible evidenciar en la figura 7 que la curva suavizada de exposición de riesgo en el período bipartidista es mayor a la curva de exposición de riesgo en el período multipartidista. Además, se exponen con más claridad los períodos de relativa estabilidad ministerial a los días 500 y 900, en la que se observa que la exposición al riesgo es igual tanto para el período pre-Constitución de 1991 como pos-Constitución de 1991.

Figura 7. Curvas Smoothed Ratio Hazard (desagregadas por períodos de estudio)

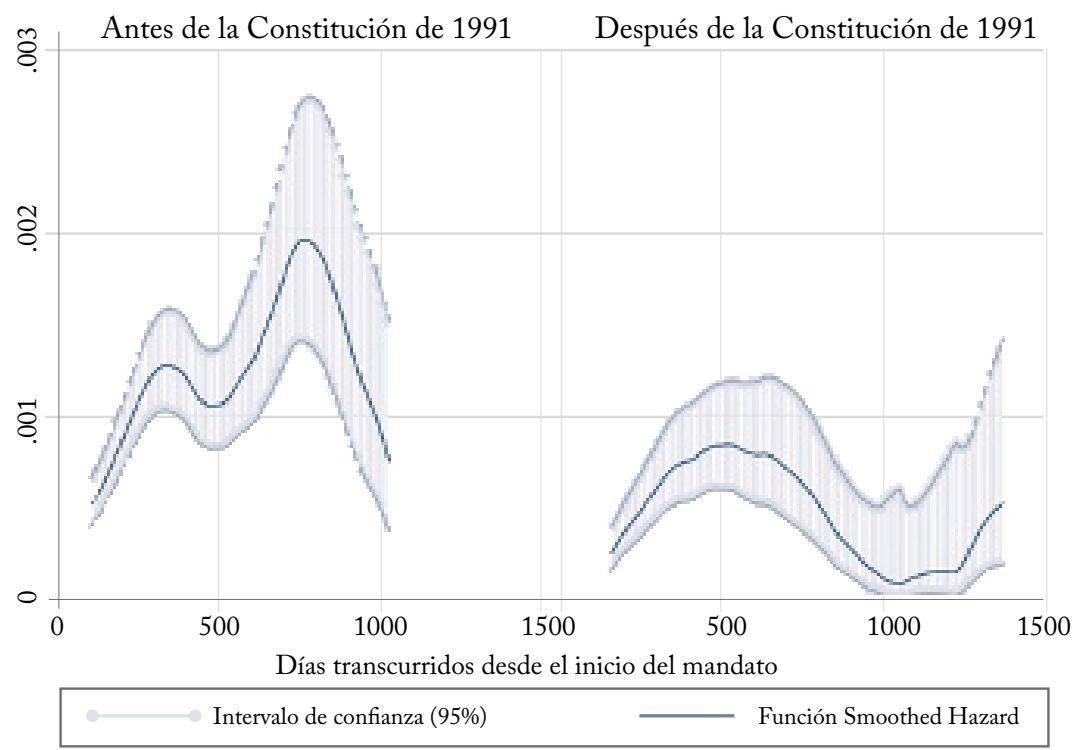

Fuente: elaboración de los autores.

2 La tasa de incidencia corresponde al número de casos que salen del gabinete con base en la población en riesgo por unidad de tiempo. 


\section{4 / Luis Bernardo Mejía Guinand - Andrés Felipe Barinas Forero - Michelle Lorena Mora Oviedo}

Ahora que ya se expusieron las diferencias significativas en la rotación ministerial a razón de variables institucionales, es fundamental analizar las características individuales de los ministros que explican un mayor o menor grado de rotación. Para ello se empleará un modelo de regresión de Cox $(1972,1975)$. En lo que respecta a los modelos de regresión de Cox, los efectos de las covariables se pueden interpretar directamente por medio de los coeficientes de riesgo o hazard ratio, los cuales están consignados en la tabla 4 . Cuando el coeficiente es menor a 1, la variable se configura como un predictor de supervivencia, dado que disminuye la probabilidad de fracaso; en una curva de supervivencia, se representaría como un desplazamiento hacia la derecha. Por el contrario, cuando el coeficiente de riesgo es mayor a 1, la variable constituye un factor de riesgo, ya que aumenta las probabilidades de ser removido del cargo durante la administración presidencial.

Tabla 4. Modelos de riesgos proporcionales de Cox con método Efron

\begin{tabular}{|c|c|c|}
\hline Variables & $\begin{array}{c}\text { (1) } \\
\text { Antes de la Constitución } \\
\text { de } 1991\end{array}$ & $\begin{array}{c}\text { (2) } \\
\text { Después de la Constitución } \\
\text { de } 1991\end{array}$ \\
\hline \multirow{2}{*}{ Carteras relevantes } & 0,789711 & $0,50704 *$ \\
\hline & $(0,1801)$ & $(0,1939)$ \\
\hline \multirow{2}{*}{ Vínculo familiar } & 0,93783 & 0,79952 \\
\hline & $(0,2940)$ & $(0,2470)$ \\
\hline \multirow{2}{*}{$\begin{array}{l}\text { Proximidad a la siguiente } \\
\text { elección }\end{array}$} & $1,000406^{*}$ & 1,0002 \\
\hline & $(0,000245)$ & $(0,00030)$ \\
\hline \multirow{2}{*}{ Maestría o PhD } & 1,3531 & 0,81540 \\
\hline & $(0,3051)$ & $(0,2333)$ \\
\hline \multirow{2}{*}{$\begin{array}{l}\text { Mismo partido del } \\
\text { presidente }\end{array}$} & $0,68633^{* *}$ & $2,1598^{* *}$ \\
\hline & $(0,1349)$ & $(0,7332)$ \\
\hline \multirow{2}{*}{ Sexo } & 2,75477 & 0,95025 \\
\hline & $(2,0174)$ & $(0,2936)$ \\
\hline \multirow{2}{*}{ Viceministro } & 0,74772 & 1,09127 \\
\hline & $(0,2689)$ & $(0,3544)$ \\
\hline
\end{tabular}




\begin{tabular}{|l|c|c|}
\hline \multirow{2}{*}{ Variables } & $\begin{array}{c}(\mathbf{1}) \\
\text { Antes de la Constitución } \\
\text { de 1991 }\end{array}$ & $\begin{array}{c}\text { (2) } \\
\text { Después de la Constitución } \\
\text { de 1991 }\end{array}$ \\
\hline \multirow{2}{*}{ Región Andina } & $0,66201 * *$ & 0,83359 \\
\cline { 2 - 3 } & $(0,1193)$ & $(0,2414)$ \\
\hline \multirow{2}{*}{ Voto popular } & $1,35116^{*}$ & 0,75037 \\
\hline \multirow{2}{*}{ Mayoría en el Senado } & $(0,2396)$ & $(0,2457)$ \\
\cline { 2 - 3 } & $1,7313 * * *$ & $0,1450^{* * *}$ \\
\hline \multirow{2}{*}{ Trayectoria pública } & $(0,3515)$ & $(0,0892)$ \\
\cline { 2 - 4 } & 1,4969 & 1,40373 \\
\hline \multirow{2}{*}{ Gobierno de coalición } & $(0,5307)$ & $(0,7001)$ \\
\cline { 2 - 3 } & $1,9679 * * *$ & $0,1576 * * *$ \\
\hline Observaciones & $(0,4034)$ & $(0,0751)$ \\
\hline Número de grupos & 314 & 205 \\
\hline
\end{tabular}

Errores estándar en paréntesis.

$* \mathrm{p}<0,1$.

$* * \mathrm{p}<0,05$.

$* * * \mathrm{p}<0,01$

Es fundamental aclarar que, pese a que el modelo estadístico por estimar es el mismo para los dos períodos, el conjunto de datos es distinto. El primer modelo se estima con los datos de los ministros que ocuparon el cargo desde 1958 hasta 1991. El segundo modelo se estima con los datos de los ministros que ocuparon el cargo desde 1991 hasta 2018. Por este motivo, hacemos la distinción en dos modelos para tener claridad sobre los efectos previos a la Constitución y los efectos luego de esta. Para el período anterior a la promulgación de la Constitución de 1991 (es decir, el modelo 1), de las 12 variables incluidas, 6 resultaron significativas: la proximidad a la siguiente elección presidencial, si el ministro pertenece al mismo partido político del presidente, si el ministro nació en la región Andina, si el ministro había ejercido algún cargo de elección popular, si el partido del ministro cuenta con mayorías en el Senado de la República y el tipo de gobierno. Por su parte, en el modelo correspondiente al período posterior a 1991 solamente 4 variables son significativas: si el ministro dirige una cartera relevante, si el ministro pertenece al mismo partido del presidente, si el partido 


\section{6 / Luis Bernardo Mejía Guinand - Andrés Felipe Barinas Forero - Michelle LoRena Mora OViedo}

del ministro cuenta con mayorías en el Senado de la República y el tipo de gobierno.

En el modelo 1, las covariables que tienen una mayor probabilidad de fracaso (constituyen factores de riesgo de salida) son: la proximidad a la siguiente elección presidencial, haciendo que conforme pasen los días para la siguiente elección se incremente el riesgo de ser removido; si el ministro había ejercido algún cargo de elección popular antes, también genera un incremento la probabilidad de fracaso; si el partido del ministro tiene mayorías en el Senado, se aumenta el riesgo de remoción, esto puede deberse a que un partido con mayorías en el Congreso tiende a estar igualmente representado como una mayoría en el gabinete, así el presidente está dispuesto a remover un ministro de dicha mayoría por tratar de consolidar el apoyo de otras fuerzas dentro del Congreso. Pese a esto, la hipótesis aún no está confirmada. Igualmente, ministros en gobiernos de coalición incrementan su probabilidad de salir del gabinete, esto puede ser producto de consolidación de mayorías y búsqueda de apoyo de otros partidos fuera de la coalición actual.

Por el contrario, las variables referentes a si el ministro pertenece al mismo partido político del presidente y si el ministro es de la región Andina tienen cocientes de riesgo menores a 1. En este sentido, los ministros con estas características tienen una menor probabilidad de ser removidos. Específicamente, los presidentes privilegian la posición de ministros de su mismo partido para dar representatividad al colectivo que apoyó su candidatura al inicio del mandato y también tienden a proteger la presencia de ministros oriundos de la región Andina.

En cuanto a los resultados del modelo 2, solamente la variable respecto a si el ministro pertenece al mismo partido del presidente presenta una tasa de riesgo mayor a 1, de tal forma que para el período entre 1991 y 2018 un ministro del mismo partido político que el presidente aumenta su probabilidad de fracaso. Esto puede deberse a la necesidad de formar gobiernos de coalición en una arena política mucho más 
diversa y competida. Contrario a lo que sucede con las variables referentes a si el ministro dirige una cartera relevante, la cual disminuye el riesgo. Otro factor de supervivencia es si el partido del ministro cuenta con mayorías en el Senado de la República, característica que reduce el riesgo de ser removido. El mecanismo explicativo de esta última variable es la constante búsqueda de estabilidad de la coalición en el legislativo, sumado al hecho de que los costos de negociación de reemplazar a un ministro con mayorías en el Congreso pueden ser altos en términos costo-efectivos, puesto que se renuncia a un apoyo político en aras de consolidar otros. Por último, si el ministro pertenece a un gobierno de coalición, la probabilidad de fracasar se reduce.

Finalmente, se examina la bondad de ajuste de los dos modelos de regresión de Cox a través de la estimación y contraste de la función de riesgo acumulada de Nelson-Aalen respecto a los residuos CoxSnell. Se considera que un modelo se encuentra ajustado cuando la diferencia entre la función de riesgo acumulada y la función de los residuos de Cox-Snell tiende a 0. Las figuras evidencian que la función de riesgo sigue muy de cerca los residuos, excepto en los valores de tiempo más grandes. Es común que los modelos con datos censurados tengan algunas fluctuaciones en grandes valores de tiempo y no es algo que cause preocupación. En general, se puede concluir que ambos modelos estimados se ajustan bien a los datos, como se observa en la figura 8 . 
28 / Luis Bernardo Mejía Guinand - Andrés Felipe Barinas Forero Michelle Lorena Mora OViedo

Figura 8. Análisis de bondad de ajuste (residuos de Cox-Snell)

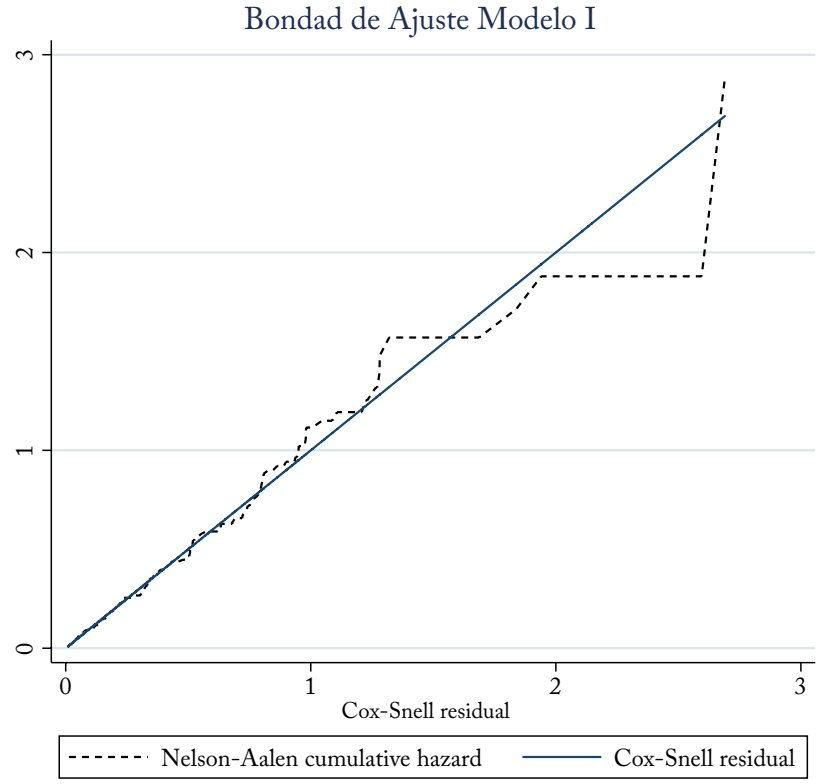

Bondad de Ajuste Modelo II

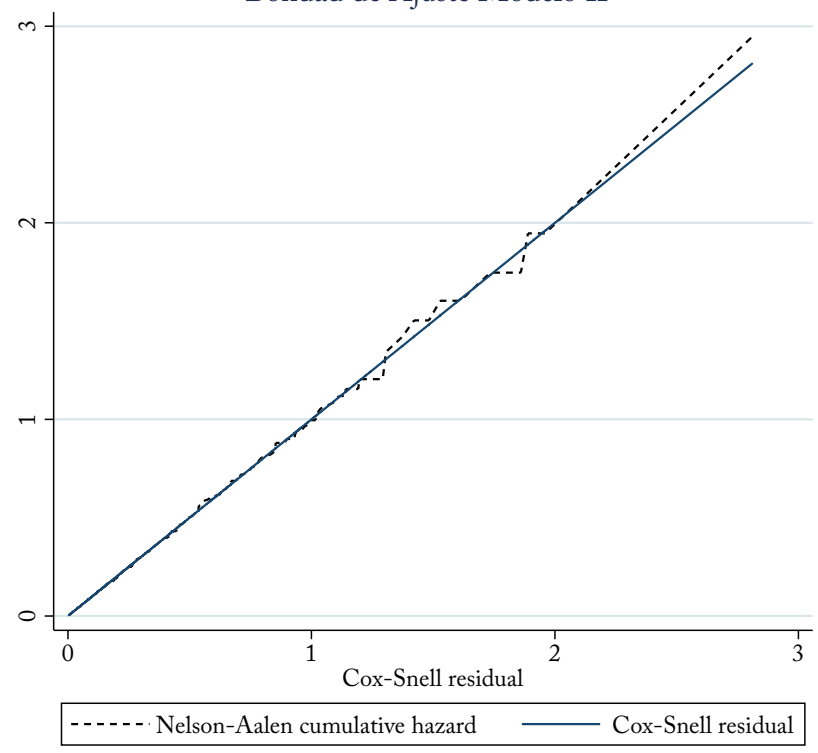

Fuente: elaboración de los autores. 
Con esto, los resultados demuestran que luego de la apertura constitucional en 1991 se dio un aumento en el número de partidos políticos que forzó a que los nuevos gobiernos colombianos tendieran a la construcción de coaliciones de gobierno. Estas coaliciones de gobierno tienen un efecto directo sobre la duración de los ministros en el cargo, haciendo que la probabilidad de ser removidos antes de la finalización del mandato sea menor a la que existía durante los períodos con sistemas bipartidistas.

\section{Conclusiones}

Si bien el análisis presentado, para el caso colombiano, muestra que las características individuales de los ministros son importantes al momento de explicar la duración de estos en el gabinete, son las características institucionales del sistema las que más inciden en su estabilidad. Para el período anterior a la promulgación de la Constitución de 1991, las variables que facilitan la permanencia de los ministros en su cargo están relacionadas con la congruencia entre el partido del ministro y el contingente legislativo de ese partido en el Senado. También se correlaciona la proximidad a la siguiente elección presidencial, la pertenencia al mismo partido político del presidente, la procedencia del ministro de la región Andina y el que el ministro hubiese ejercido algún cargo de elección popular. Después de 1991, período en el cual el sistema de partidos se caracteriza como multipartidista, la permanencia de los ministros en sus cargos puede estar influida por variables como si el gobierno es o no de coalición, si el ministro pertenece al mismo partido del presidente y si el partido del ministro cuenta con mayorías en el Senado. Variables como el sexo, el vínculo familiar o el nivel educativo del ministro no reportan relevancia al momento de explicar el tiempo de duración en sus cargos en ninguno de los dos sistemas analizados.

El estudio igualmente sugiere que la estabilidad en los cargos de los ministros en sistemas bipartidistas es menor que la duración de los ministros en presidencialismos de coalición. En particular, este trabajo evidencia que existe una mayor estabilidad en el gabinete ministerial 


\section{0 / Luis Bernardo Mejía Guinand - Andrés Felipe Barinas Forero - Michelle Lorena Mora Oviedo}

después de la promulgación de la Constitución de 1991 respecto al período previo comprendido entre 1958 y 1994. Durante el período bipartidista solo el 30\% de los ministros duraban más de 1000 días en el cargo, mientras que en el período posterior a 1991 se observa una tasa de supervivencia cercana al 60\%. Esto puede explicarse por el hecho de que cuando la arena política es más competitiva, y el número efectivo de partidos crece, las coaliciones de gobierno aparecen como necesarias. Sin embargo, entre mayor es el número de partidos que hace parte de la coalición, mayores son los costos de negociación en los que incurre el presidente y, por lo tanto, menores son los incentivos para cambiar su gabinete cuando enfrenta eventos críticos. En otras palabras, el multipartidismo incentiva la estabilidad de los ministros.

La agenda de investigación hacia el futuro debe entonces buscar comprender cómo funciona la negociación entre el presidente, los partidos y los candidatos a ministros al momento de negociar el ingreso o la salida del gabinete. En especial, es relevante entender cuáles son los costos de esta negociación y avanzar en su operacionalización empírica.

\section{Referencias}

Albala, A. (2016). Presidencialismo y coaliciones de gobierno en América Latina: un análisis del papel de las instituciones. Revista de Ciencia Política, 36(2), 459-479. https://doi.org/10.4067/S0718-090X2016000200003

Amorim Neto, O. (1998). Cabinet formation in presidential regimes: An analysis of 10 Latin American countries. Rio de Janeiro: Instituto Universitário de Pesquisas do Rio de Janeiro (IUPERJ).

Amorim Neto, O. (2006). The Presidential Calculus: Executive Policy-making and Cabinet Formation in the Americas. Comparative Political Studies, 39(4), 415-440. https://doi.org/10.1177/0010414005282381

Arana Araya, I. (2012). ¿Quién le susurra al presidente? Asesores vs. ministros en América Latina. Revista de Ciencia Política, 50(2), 36-61. https:// doi.org/10.5354/0716-1077.2013.25868 
Archer, R., \& Shugart, M. (1997). The unrealized potential of presidential dominance in Colombia. In S. Mainwaring \& M. Shugart (Eds.), Presidentialism and democracy in Latin America (pp. 110-159). New York: Cambridge University Press.

Camerlo, M., \& Pérez Liñán, A. (2015). Minister Turnover, Critical Events, and the Electoral Calendar in Presidential Democracies. The Journal of Politics, 77(3), 608-619. https://doi.org/10.1086/681028

Cárdenas, M., \& Pachón, M. (2010). Cómo la Constitución de 1991 cambió los procesos de formulación de políticas públicas. En M. Cárdenas, C. Scartascini \& E. Lora (Eds.), Consecuencias imprevistas de la Constitución de 1991: la influencia de la política en las políticas económicas. Bogotá: Fedesarrollo-Alfaomega.

Chasquetti, D. (2001). Democracia, multipartidismo y coaliciones en América Latina: evaluando la difícil combinación. En J. Lanzaro \& M. Novaro (Eds.), Tipos de presidencialismo y coaliciones politicas en América Latina (pp. 319-359). Buenos Aires: Clacso.

Chasquetti, D. (2008). Democracia, presidencialismo y partidos politicos en América Latina: evaluando la "difícil combinación”. Montevideo: Cauce Editorial. Chasquetti, D., Buquet, D., \& Cardarello, A. (2013). La designación de gabinetes en Uruguay: estrategia legislativa, jerarquía de los ministerios y afiliación partidaria de los ministros. América Latina Hoy, (64), 15-40. https://doi.org/10.14201/alh.10230

Cheibub, J. (2006). Presidentialism, parliamentarism and democracy. Cambridge: Cambridge University Press.

Cox, D. (1972). Regression models and life-tables. Journal of the Royal Statistical Society Series B (Methodological), 34(2), 187-220.

Cox, D. (1975). Partial likelihood. Biometrika, 62(2), 269-276.

Efron, B. (1977). The efficiency of Cox's likelihood function for censored data. Journal of American Statistical Association, 72(359), 557-565.

Figueiredo, A., \& Limongi, F. (2008). Instituciones políticas y gobernabilidad: desempeño del gobierno y apoyo legislativo en la democracia brasileña. En C. Melo \& M. Alcántara (Eds.), La democracia brasileña. Balance y perspectivas para el siglo 21 (pp. 117-156). Salamanca: Ediciones Universidad de Salamanca.

González Bustamante, B., \& Olivares, A. (2016). Cambios de gabinete y supervivencia de los ministros en Chile durante los gobiernos de la 


\section{I Luis Bernardo Mejía Guinand - Andrés Felipe Barinas Forero - Michelle Lorena Mora OViedo}

Concertación (1990-2010). Colombia Internacional, 87, 81-108. https:// doi.org/10.7440/colombiaint87.2016.04

Hartlyn, J. (1988). The politics of coalitional rule in Colombia. Cambridge: Cambridge University Press.

Jones, M. (2005). The role of parties and party systems in the policymaking process. Prepared for the workshop on State reform, public policies, and policymaking processes. Washington, D.C.: Inter-American Development Bank

Kaplan, E., \& Meier, P. (1958). Nonparametric estimation from incomplete observations. Journal of the American Statistical Association, 53(282), 457-481. https://doi.org/10.2307/2281868

Lanzaro, J. (2001). Tipos de presidencialismo y coaliciones políticas en América Latina. Buenos Aires: Clacso.

Martínez Gallardo, C. (2014). Designing cabinets: presidential politics and ministerial instability. Journal of Politics in Latin America, 6(2), 3-38.

Mainwaring, S. (1990). Presidentialism in Latin America. Latin America Research Review, 25(1), 157-179.

Mainwaring, S. (1992). Dilemmas of multiparty presidential democracy: the case of Brazil. Working Paper No 174. Notre Dame: May Kellogg Institute.

Mejía Guinand, L. B., \& Botero, F. (2019). PresCab_Basic_Colombia (2019) Presidential Cabinets Project. https://doi.org/10.7910/DVN/QPC6IV

Ollier, M., \& Palumbo, P. (2016). ¿Caso testigo o caso único? Patrones de la formación de gabinete en el presidencialismo argentino (19832015). Colombia Internacional, 87, 53-80. https://doi.org/10.7440/ colombiaint87.2016.03

Payne, M. (2007). Party systems and democratic governability. In M. Payne, D. Zovatto, F. Carrillo \& A. Allamand (Eds.), Democracies in development politics and reform in Latin America (pp. 127-155). Washington, D.C.: Banco Interamericano de Desarrollo. 